\title{
Dynamic Random Number Generator based on User Seed(s)
}

\author{
Saleh N. Abdullah, Ph.D \\ Assistant Prof., Khawlan College, Sana'a University, \\ Yemen.
}

\author{
Sharaf A. Alhomdy, Ph.D \\ Assistant Prof. \& Vice-Dean, Faculty of Computer \\ and Information Technology, Sana'a \\ University, Yemen.
}

\begin{abstract}
Random number generators (RNGs) is an underlying technology to accomplish highly secure systems. Therefore, for any security or simulation, systems should be associated with RNGs. Many of RNGs are currently in use, but the main defects in the available RNGs are the short period of its repeat cycle length and the predefined values of static factors as well. In this paper, we will try to suggest a method to extend the periodic cycle of the repetition and to use dynamic factors instead of static factors based on the seed values for the sake of security enhancement.
\end{abstract}

\section{Keywords}

Seed, Period, Static Factors, Dynamic Factors, RNGs, Security, Simulation, LCM, Linear Congruential, Uniformity, Independence.

\section{INTRODUCTION}

The random number generators (RNGs) are useful for a variety of purposes, such as [1]:

a) Generating data encryption keys.

b) Simulating and modeling complex phenomena.

c) Selecting random samples from larger data sets.

d) Testing problem generation for the performance evaluation of computer algorithms.

e) Statistically sampling, and so on.

Moreover, nowadays, many of daily activities in security need RNGs to perform their tasks. For example, secret and public-key generation and challenge-response authentication require unpredictable random numbers. Therefore, despite the large amount of theoretical research already done on this subject, many of the generators currently in use, for example the RNGs are an underlying technology to accomplish highly secure systems. But the main defects in the available RNGs are $[1,2]$ :

The short periods of its repeat cycle length.

1) Sometimes such available RNGs do not satisfy the desire needs for specific applications.

2) The predefined values of static factors may reduce the associated security.

In this paper, we will try to suggest a new method to extend the periods of repeat cycle length to generate random numbers. The method can be used for the specific applications by determining the number of digits. It also uses dynamic factors based on the seed value(s) to enhance the associated security. The paper has been organized in a flexible manner. Section II focuses on the background related to the previous work. Section III explains a proposal method to enhance the security produced. Section IV discusses the analysis \& complexity for the proposed method and the experimental result shown in section IV. Conclusion \& future research assignments will be highlighted in section V.

\section{BACKGROUND}

Indeed, all RNGs are based upon specific mathematical algorithms, which are repeatable and sequential. As such, it would be satisfying to generate random numbers from a process that is according to well-established understanding. So, to be useful in simulation, a sequence of random numbers $R_{1}, R_{2}$ must have two important properties: uniformity and independence. That is, each random number $R_{i}$ is an independent sample drawn from a continuous uniform distribution between 0 and 1 (mean $1 / 2$, standard deviation). Some consequences of the uniformity and independence properties are as follows [2,3]:

a) Uniformity: if the interval $[0,1]$ is divided into $n$ sub-intervals of equal length, the expected number of observations in each interval $\mathrm{N} / \mathrm{n}$, where $\mathrm{N}$ is the total number of observations. The distribution of numbers in the sequence should be uniform; that is the frequency of occurrence of each number should be the same.

b) Independence: the probability of observing a value in a particular interval is independent of the previous values drawn. This means that no value in the sequence can be inferred from the others.

Mainly there are many methods used to generate random numbers. In the most methods the modulus (m) should be as large as possible, because a small set of numbers make the outcome easier to predict. We will mention some of them as follows. [2, 4, $5,6]$ :

\subsection{Linear Congruential Method (LCM)}

This method is used to generate a sequence of integers $X_{1}$, $\mathrm{X}_{2}, \ldots \mathrm{X}_{\mathrm{n}}$ between 0 and $\mathrm{m}-1$ followed by a recursive relationship as in Eq. (1):

$$
X_{i}=\left(a X_{i-1}+c\right) \bmod m
$$

Such that the parameters as:

$$
\begin{aligned}
& \mathrm{X}_{0}=\text { seed (or starting value) } \\
& \mathrm{m}=\text { modulus(or divisor) } \\
& \mathrm{a}=\text { multiplier } \\
& \mathrm{c}=\text { increment }
\end{aligned}
$$

Where $\mathrm{m}>0$ and $\mathrm{a}<\mathrm{m}, \mathrm{c}<\mathrm{m}, \mathrm{X}_{0}<\mathrm{m}$

The selection of the values for a, c, m, and $\mathrm{X}_{0}$ drastically affects the statistical properties and the cycle length. The random integers $X_{i}$ are being generated in the interval [0,m-1]. The random numbers generated are then calculated as in Eq. (2):

$$
R_{i}=\frac{X_{i}}{m}, \text { for } \quad i=1,2, \ldots, k
$$

Therefore, the criteria of good RNG is to satisfy maximum density and it leaves no large gaps on $[0, \mathrm{~m}-1]$. Such that maximum density means that all numbers between $[0, \mathrm{~m}-1]$ 
should appear in predictable manner. According to [2] to achieve the maximum density and avoid cycling we have to choose the suitable values for the factors $\mathrm{a}, \mathrm{c}, \mathrm{m}$ and $\mathrm{X}_{0}$.

There are main deficiencies in the LCM, if an opponent knows that LCM is being used and if the parameters are known, and once a single number is discovered, then all subsequent numbers can be easy to know. Even if the opponent knows only that LCM is being used, then the knowledge of a small part of sequence is sufficient to determine the parameters of the algorithm. Suppose that the opponent is able to determine values for $\mathrm{X}_{0}, \mathrm{X}_{1}, \mathrm{X}_{2}$, and $\mathrm{X}_{3}$. Then

$$
\begin{aligned}
& X_{1}=\left(a X_{0}+c\right) \bmod m \\
& X_{2}=\left(a X_{1}+c\right) \bmod m \\
& X_{3}=\left(a X_{2}+c\right) \bmod m
\end{aligned}
$$

Those equations (3), (4) and (5) can be solved to get the parameters $\mathrm{a}, \mathrm{c}$, and $\mathrm{m}$.

\subsection{Combined Linear Congruential Generators}

This method obtains the longer period generator because it combines two or more multiplicative congruential generators [2, 7].

- $\quad$ Let $\mathrm{X}_{\mathrm{i}, 1}, \mathrm{X}_{\mathrm{i}, 2}, \ldots, \mathrm{X}_{\mathrm{i}, \mathrm{k}}$ be the $\mathrm{i}^{\text {th }}$ output from $\mathrm{k}$ different multiplicative congruential generators.

- $\quad$ The $\mathrm{j}^{\text {th }}$ generator $\mathrm{X}_{0, \mathrm{j}}$ :

- $\quad X_{i+1, j}=\left(a_{i} X_{i}+c_{i}\right) \bmod m_{i}$

- Such that $m_{j}$ is a prime modulus, $a_{j}$ is multiplier, and $m_{j}-1$ is a period.

- $\quad$ Produces integers $\mathrm{X}_{\mathrm{i}, \mathrm{j}}$ approximate $\sim$ Uniform in $\left[0, \mathrm{~m}_{\mathrm{j}}-\right.$ $1]$.

- $W_{i, j}=X_{i, j-1}$ approximate $\sim$ Uniform on integers in [0, $\left.\mathrm{m}_{\mathrm{j}}-2\right]$.

$$
X_{i}=\left(\sum_{j=1}^{k}(-1)^{j-1} X_{i, j}\right) \bmod m_{i}-1
$$

Then the maximum possible period shown in Eq (8) as follows:

$$
P=\frac{\left(m_{1}-1\right)\left(m_{2}-1\right) \ldots\left(m_{k}-1\right)}{2^{k-1}}
$$

\subsection{RNG using Cipher Text}

This method uses any cipher text to generate random numbers by converting the cipher text to binary digits and selects suitable numbers of binary digits to be converted to decimal digits. But the main defects are that it needs more calculation [3].

\section{PROPOSED METHOD}

The main idea of the suggested method is to use combined linear congruential generators based on generating the parameters $a_{i}, c_{i}$, and $\mathrm{m}_{\mathrm{i}}$ dynamically depending on the user seed(s) named as $\left(\mathrm{X}_{0, \mathrm{j}}\right)$. It also uses the concatenation of several generators numbers dynamically (CONCATENAT $\left(X_{i}\right)$ such that $\left.i=1, . . k\right)$. Hence, started point $\mathrm{i}$ changes periodically.

\section{2- Compute}

$$
a_{j}=\operatorname{geta}\left(X_{0, j}+n\right)
$$

where $n$ is the number required $\& a_{j}$ is prime

number $\approx=1+4 k$, and $k$ is an integer.

\section{3- Compute the}

$$
c_{j}=\text { prime }\left(a_{j}\right)
$$

4- Compute

$m_{j}=\operatorname{getm}\left(a_{j}+n\right), m_{j}=2^{b}$

such that $b$ is integer number.

5- For $\mathrm{j}=1$ to $k$,

$$
\begin{aligned}
& X_{j}=\left(a_{j} X_{i, j}+c_{j}\right) \bmod m_{j} \\
& X_{i+1, j}=X_{j}
\end{aligned}
$$

where $\mathbf{i}=0$ to $\mathrm{n}$, and $\mathrm{k}$ is an integer number depends on security required.

6- Compute

$$
X_{i+1}=\text { CONCATENATE }\left(X_{j}\right) \text { for } k \text { 's values, }
$$

such that $\mathbf{j}$ position change dynamically.

7- Set $i=i+1$

8- If $i<=n$ go to step 5.

9- Stop.

4. ANALYSIS AND COMPLEXITY STUDY In linear congruential method, the Eq. (1) is used to generate the RNGs. Thus the selection of the values for the parameters a, c, and $\mathrm{m}$ drastically affect the statistical properties and the cycle length.

Meanwhile, in combined linear congruential generators, using the Eq. (6) with the same parameters a, c, and $m$ which are used as a partial part and the selection of the values for the parameters drastically affects the statistical properties and the cycle length.

Whereas, the concatenation or addition of two or more random numbers is a method to produce a new random number with the same statistical properties and to increase the cycle length. The time complexity to concatenate two or more random numbers always less than the addition of the same numbers. Therefore, it can be said that the time complexity is less than the current combined method.

\section{EXPERIMENTAL RESULTS}

This section explains some examples for experimental results for different essential seeds using our generated simulation software according to the proposed algorithm mentioned in section III. As a result, three different experiments have been done with different user seeds, it gives different outputs. The result shows that, there are no reputation value and the cycle period is better than the other algorithms. Table (1) shows the result for 50 values using four essential seeds $(12,129,512,985)$. Table (2) shows the result for 50 values using four essential seeds $(55,278$, 985, 1299); whereas, Table (3) shows the result for 50 values using four essential seeds $(980,1200,2440,3600)$.

The algorithm becomes as follows:

1- Select seeds $\left(X_{0, j}\right)$ such that $\mathrm{j}=1$ to $\mathrm{k}$. 
Table (1): Data Generated for 4 values of Essential Seeds (12, $129,512,985) \& 50$ Output

\begin{tabular}{|l|l|l|}
\hline Generated value & Generated value & Generated value \\
\hline 9723257448 & 3223719403657 & 2910812053664 \\
\hline 845710962745 & 5714411692272 & 4035713082345 \\
\hline 53841741368 & 2836918082457 & 171846172048 \\
\hline 4834918283081 & 7718816373216 & 10041600121 \\
\hline 1053524493872 & 5621314521769 & 1014208934016 \\
\hline 449715683929 & 651366653648 & 644611802505 \\
\hline 12526815572768 & 116256161593 & 254812651376 \\
\hline 7269601193 & 2124415813568 & 6033718401305 \\
\hline 113882011152 & 806110921929 & 453489652320 \\
\hline 4911443065 & 7325615692976 & 884371228617 \\
\hline 696817573120 & 76655762777 & 334012732752 \\
\hline 961734681353 & 9342819091872 & 20505136441 \\
\hline 1214641361480 & 10429386041 & 11740414212672 \\
\hline 923051872153 & 815041833256 & 112285356777 \\
\hline 135082931424 & 3648916881913 & 411606412080 \\
\hline 12014910043561 & 372285732224 & 1083316321625 \\
\hline 145618811856 & 37011777342051 & \\
\hline
\end{tabular}

Table (2): Data Generated for 4 values of Essential Seeds (55, $278,985,1299) \& 50$ Output.

\begin{tabular}{|l|l|l|}
\hline Generated value & Generated value & Generated value \\
\hline 1061954483924 & 1873703657741 & 15095136642246 \\
\hline 3175027452551 & 9877922723960 & 991382345585 \\
\hline 2069593682922 & 1353262457987 & 21872320481180 \\
\hline 1393430811709 & 1343273216782 & 11130121703 \\
\hline 2102738723776 & 5158176917 & 1907940164018 \\
\hline 21588639291699 & 7473936483556 & 9121025053701 \\
\hline 1182152768342 & 19184615933975 & 668111376520 \\
\hline 34901193473 & 17473535682042 & 3993413053435 \\
\hline 18624311522860 & 4389819292621 & 1026152320926 \\
\hline 15126306579 & 1785929762384 & 2114106171953 \\
\hline 15887931201090 & 11947027772099 & 4225927523188 \\
\hline 2515013532565 & 8650318722534 & 959424411303 \\
\hline 348434801176 & 16384241361 & 14251126721162 \\
\hline 1995181531787 & 154787256444 & 2037387773533 \\
\hline 7090314241070 & 17522219133551 & 146912080992 \\
\hline 11576235613889 & 12665522242258 & 235416252499 \\
\hline
\end{tabular}


[5] Figiel, K.D. and Sule. D.R. 1985. "New Lagged Product Test for Random Number Generators", Comput. Ind. Eng. Vol. 9, 287-296.

[6] S. Japertas and et al. 2007. "Unpredictable Cryptographic Pseudo-Random Number Generator based on Non-linear Dynamic Chaotic System", Electronics and Electrical Engineering ISSN $1392-1215$, pp 29 -32.

[7] P. L'Ecuyer 1988. "Efficient and Portable Combined Random Number Generators", Communications of the ACM 31 June 1988, Volume 31 No. 6, USA.

[8] Douglas W. Mitchell 1993. "A Nonlinear Random Number Generator with Known, Long Cycle Length", Cryptologia, Volume 17 Issue 1, pp 55- 62, USA.

\section{AUTHOR's PROFILE}

Dr. Saleh Noman Abdullah Alasaly, born in 1969, Gabel Habashee, Taiz, Republic of Yemen. Ph.D. in Infromation Securty, SRTMU, India, 2005. Assistant Prof., Khawlan College, Sana'a University, Yemen.

E-mail: saleh.alasali97@yahoo.com

Dr. Sharaf A. Alhomdy, born in 20/01/1971, Alsena, Taiz, Republic of Yemen. Ph.D. in Computer Scince, Pune University, India, 2009. Asst. Prof. \& Vice-Dean, Faculty of Computer and Information Technology, Sana'a University, Yemen.

E-mail: sharafalhomdy@gmail.com. 